\section{Blood Pressure and the Pill}

The administration of oral contraceptives results in a statistically significant rise in the level of the blood pressure. ${ }^{12}$ But doubt remains how often this rise is clinically significant, and further, whether a subgroup of women can be identified who are specially liable to this complication.

The original reports of hypertension in women taking oral contraceptives were retrospective and on small numbers of women. ${ }^{3}$ Sporadic case reports even showed that malignant hypertension might result. ${ }^{5}$ One of the best planned prospective studies, incorporating an adequate control group, showed a significant rise in systolic but not diastolic pressure in women given these agents. It also showed that in none of the women studied did the level of blood pressure exceed $140 / 90 \mathrm{~mm} \mathrm{Hg}$ while taking the medication. ${ }^{1}$ In this and other studies ${ }^{6}$ there were variations between patients in the time the drug was administered before hypertension developed and also in the time taken for the pressure to fall when they were withdrawn.

Two recent reports examine some other aspects of the problem. In a prospective study from South Australia ${ }^{7}$ of 74 women the administration of oral contraceptives resulted in a significant rise $(7 \mathrm{~mm} \mathrm{Hg})$ in the mean systolic pressure; the rise in diastolic pressure $(1.8 \mathrm{~mm} \mathrm{Hg}$ ) was not significant. The time of observation of the patients ranged from three months to 2 years. In an attempt to separate "clinical" from "statistical" hypertension, cut-off points of $150 \mathrm{~mm} \mathrm{Hg}$ (systolic) and $90 \mathrm{~mm} \mathrm{Hg}$ (diastolic) were chosen. Before starting on contraceptive drugs $1.19 \%$ and $6.49 \%$ of the group had systolic and diastolic hypertension respectively; this was mainly in older and heavier patients. When contraceptives were given, 6 women $(8.1 \%)$ developed sustained systolic hypertension and 3 women (4.05\%) systolic and diastolic hypertension. Further, women with a parental history of hypertension, or a history of hypertension in pregnancy, showed greater increases in pressure. Statistically the rise in systolic pressure correlated with the age of the patientthat is, the older the patient, the more likely was the pressure to rise.

In a larger study from California ${ }^{8}$ much the same conclusions have been reached. The blood pressure was measured in 7,605 women aged between 18 and 60 years, including present users of oral contraceptives, women who had never used them, and there who had stopped. Women on antihypertensive drugs were excluded from the survey. In all age groups women on oral contraceptives had on average a higher systolic but not diastolic blood pressure. When the authors defined "clinical" hypertension as greater than 140/ $90 \mathrm{~mm} \mathrm{Hg}$, women taking oral contraceptives accounted for most of such cases. There was no difference between the blood pressures of the past users and those who have never taken the drugs. No association was found between the duration of treatment and the incidence of hypertension.

It thus appears that women more likely to develop hypertension spontaneously - that is, the older, the heavier, those with a history of hypertension in pregnancy, or a family history of the disease-are the ones more liable to a rise in blood pressure when taking oral contraceptives. The risk of severe hypertension appears to be reassuringly low. When this topic was reviewed in these columns previously 910 it was suggested that, if a woman is going to be given these agents, her blood pressure should be measured, and subsequently checked at, say, six-monthly intervals. This would appear to be especially important if she is in the older childbearing years or if she is overweight. It was further suggested that if she was already hypertensive the blood pressure should be checked more assidously. This would still seem to be reasonable advice.

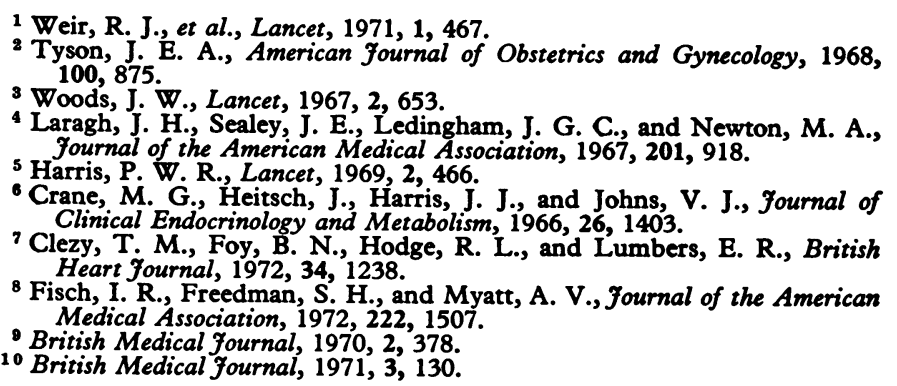

\section{Anaesthetic Contamination}

In recent years evidence has been accumulating that staff working in the operating theatre may be at special risk from contamination of the atmosphere with anaesthetic gases and vapours. E. N. Cohen and colleagues ${ }^{1}$ reported a $30 \%$ incidence of spontaneous abortion in nurses working in operating rooms compared with $9 \%$ in nurses on general duties, and they found an even more striking incidence of $38 \%$ in women anaesthetists compared with $10 \%$ for a control group of women physicians in other specialties. Other workers have reported similar findings. ${ }^{2} 3$

A 20-year survey of the causes of death in American anaesthetists disclosed a higher incidence of lymphoid and reticuloendothelial malignancies than in a comparable sample of the population, though there were other differences such as a lower incidence of bronchial carcinoma and coronary artery disease. ${ }^{4}$ Hepatitis in anaesthetists has been attributed to repeated exposure to subanaesthetic concentrations of halothane, 56 and recently ophthalmic sensitivity in the form of irritation and redness of the eyes has been reported. ${ }^{7}$ A faster rate of metabolism of halothane in anaesthetists than in pharmacists ${ }^{8}$ suggests that chronic exposure to halothane alters the body's response to the drug. Though long-term exposures of experimental animals to anaesthetic agents have demonstrated toxicity, these have been with higher concentrations than those obtaining in operating theatres, and H. W. Linde and D. L. Bruce9 found no pathological change in the organs of rats exposed to 100 parts per million (p.p.m.) halothane in air for 8 months.

C. E. Whitcher and colleagues ${ }^{10}$ measured the halothane concentration in two large operating rooms with 10 changes of air an hour and found 9 p.p.m. with non-rebreathing anaesthetic systems and 5 p.p.m. with semiclosed systems. These were associated with halothane end-tidal air concentrations of 0.2 p.p.m. in operating room nurses and 0.46 in anaesthetists, with small residual concentrations as long as 16 hours after leaving the theatre. Higher concentrations were noted by $P$. Nikki and colleagues ${ }^{11}$ in six operating rooms, most of which had natural ventilation only, and they also found 3.4 p.p.m. in adjacent recovery rooms. The use of scavenging techniques to avoid contamination of room air with expired air containing halothane vapour reduced the concentration of the drug by $90 \%$ and a similar effect was noted in the concentration of nitrous oxide in room air. But 